\title{
Travestis, marimachas y maricones: el camino del arcoíris en El Salvador
}

\author{
Travestis, marimachas and maricones: The way of the rainbow in \\ El Salvador
}

Amaral Palevi Gómez

\begin{abstract}
Resumen
Este artículo tiene el objetivo de establecer un marco histórico de la organización social de lesbianas, gays, bisexuales, transexuales y transgéneros (LGBT) en San Salvador, El Salvador. Asimismo, procura contribuir al resguardo de la memoria histórica de las minorías sexuales en El Salvador. La metodología utilizada fue la exploratoria, para recuperar la información del movimiento social y organizaciones LGBT (desde el surgimiento de las dictaduras militares en 1932 hasta el 2015). Las técnicas de investigación fueron las entrevistas a activistas LGBT y una búsqueda arqueológica documental. Los resultados apuntan a la organización social de las identidades LGBT en San Salvador y conquistas políticas restringidas.
\end{abstract}

Palabras claves: El Salvador - LGBT. Minorías sexuales - organización social - memoria histórica.

\begin{abstract}
This paper aims to establish a historical context of the social organization of lesbians, gays, bisexuals, transsexuals and transgender (LGBT) in San Salvador, El Salvador. It also seeks to contribute to safeguard the historical memory of sexual minorities in El Salvador.The methodology was exploratory to retrieve information about social movement and LGBT organizations (since the emergence of military dictatorships in 1932 through 2015). Research techniques included interviews with LGBT activists and documentation examination. The results point out to the social organization of the LGBT identities in San Salvador and their limited political gains.
\end{abstract}

Key words: El Salvador. LGBT - sexual minorities - social organization - historical memory.

Fecha de recepción: Junio 2015

Fecha de aprobación: Septiembre 2015

Doctor en Estudios Internacionales en Paz, Conflictos y Desarrollo. Sus líneas de investigación son Estudios de Paz, Violencia y Estudios LGBT. Cuenta con experiencia en la gestión de proyectos para el desarrollo comunitario y docencia. E-mail: amaral.palevi@gmail.com 


\section{INTRODUCCIÓN}

Las jerarquías de género en El Salvador se puede afirmar que se estructuran desde una mirada masculina, mestiza y urbana (Lara-Martínez, 2012: 136), que tiende a promover y reproducir la diferencia y esencialismos sexuales (Martín-Baró, 1992; Vásquez, Ibañez y Murguialday, 1996, Lara, 2013) entre hombres y mujeres, en los que se conforma el modelo de hombre blanco, profesional, cristocéntrico, casado con una mujer como lo ideal. Así colateralmente, considero que el heterosexismo organiza y jerarquiza las identidades de género. Las manifestaciones de identidades de género masculinas diferentes al modelo anterior son menospreciadas e invisibles para los discursos oficiales acerca de familia, matrimonio, educación, trabajo entre los más importantes. Lo antes expuesto propicia discriminación, violencia y muertes para las minorías sexuales. En este ambiente hostil, pese a los avances políticos y legislativos en algunos países de América Latina, sobre todo en países del Cono Sur (International Lesbian, Gay, Bisexual, Trans and Intersex Association, 2015; Barrientos, 2015), existe un movimiento social de lesbianas, gays, bisexuales e identidades trans (LGBT) que reivindica derechos al Estado y deberes a la sociedad en general.

La indagación de temas ligados a la sexualidad en El Salvador es un desafío que pocos autores se han atrevido a realizar, debido principalmente a la connotación de tabú que se le ha asignado a este tema por parte del pensamiento conservador imperante en el país. Dicho pensamiento ha tratado de ocultar el cuerpo, el género y la sexualidad de los discursos públicos, académicos y oficiales (Lara-Martínez, 2012), dando como resultado una escasa producción académica en el área de historia en general y en específico respecto de estas temáticas.

Este artículo tiene el objetivo de establecer un marco histórico de la organización social de lesbianas, gays, bisexuales, transexuales y transgéneros (LGBT) en San Salvador, El Salvador; visibilizando la existencia de sexualidades disidentes y el tratamiento que el orden sexogénero binario dominante les proporcionó entre 1932 y 2015. A la vez, busca contribuir al resguardo de la memoria histórica de las minorías sexuales en El Salvador.

La metodología utilizada fue la exploratoria, determinada por la no existencia de otros trabajos académicos que aborden la temática en cuestión, y tomando en cuenta que investigar concerniente a la historia en El Salvador es recurrir a "fuentes inéditas" (Prudhomme, 2010: 92), y más aún cuando se investiga en relación con el cuerpo, género y sexualidad, y en específico por sexualidades disidentes. Por tales motivos se recurre a producciones literarias salvadoreñas como fuente de información del "trabajo arqueológico" (Foucault, 1972) emprendido. También se procuró información en publicaciones nacionales e internacionales que contienen información del objeto de estudio o categorías para su análisis.

De igual forma se realizaron varias entrevistas a activistas LGBT organizados y responsables institucionales: Joaquín Cáceres (Asociación Salvadoreña de Derechos Humanos "Entre Amigos"), Karla Avelar (Asociación Comunicando y Capacitando a Mujeres Trans con VIH y Sida en El Salvador -Concavis-Trans-), Vi Rivera (Hombres Trans El Salvador-HT503-), Douglas Salazar ${ }^{\dagger}$ (Diversidad UES), Cruz Torres (Dirección de Diversidad Sexual, Secretaria de Inclusión Social). A todos ellos y ellas les agradezco el tiempo y la valiosa información proporcionada acerca de sus experiencias de vida, acciones institucionales y proyecciones organizacionales, que al mismo tiempo constituyen el camino del arcoíris del movimiento social LGBT.

El presente artículo se encuentra dividido en seis apartados. El primero de ellos hace referencia a lo LGBT antes de 1970, en donde se recurre a fuentes literarias para recolectar 
información. El segundo apartado versa respecto de las acciones de represión política en la década de 1970 y el surgimiento de la primera discoteca gay denominada Oráculos en San Salvador. En el tercer apartado nos adentramos a las acciones de la Guerra Interna de la década de 1980, recurriendo nuevamente a fuentes literarias para recuperar referencias de la existencia de personas LGBT en los frentes de guerra del Ejército o del Frente Farabundo Martí para la Liberación Nacional (FMLN). En el cuarto apartado, en el periodo de la posguerra, surge lo que podemos denominar como movimiento LGBT salvadoreño por medio de la organización de dos grupos gay, uno lésbico y uno trans en paralelo. En el quinto apartado se narra el proceso de diversificación del movimiento y la constitución de varias organizaciones LGBT. En el sexto apartado se habla de una internacionalización del movimiento y algunas conquistas políticas restringidas.

\section{ANTECEDENTES HISTÓRICOS DE LO LGBT ANTES DE 1970}

La historia contemporánea de El Salvador está marcada por la violencia en sus más diversas formas. Para tener un referente, en 1932 se realizó la masacre de los pueblos indígenas de la zona occidental de El Salvador, conocidos como Los Izalcos, con un saldo histórico de 30.000 muertos, con la subsecuente fragmentación cultural y social de este pueblo originario (Audiovisuales UCA, 2007). Con esta masacre se inauguran las dictaduras militares en el país por más de 50 años.

Antes de la realización de este genocidio el alemán Leonhard Schultze-Jena en 1930, en tres meses de intenso trabajo, transcribió más de 50 relatos de mitos en lengua náhuat-pipil. Lara-Martínez (2012), quien tradujo e interpretó dichos textos, nos presenta una traducción de un relato recuperado por Schultze-Jena que habla de la sexualidad, y en específico el acto sexual entre dos hombres en la cultura Náhuat-Pipil². El relato se llama La boda del vagabundo (Lara-Martínez, 2012: 84-86). En este relato se presenta un acto de penetración sexual de un hombre mayor por parte de otro más joven que le ganó una apuesta para casarse con su hija y poseerlo antes de la boda con ella. El acto sexual descrito en ningún momento se puede catalogar como homosexual.

Siguiendo el argumento de Bourdieu (1999) acerca de la libido dominandi, como la mayor expresión de la dominación masculina, considero que la orientación sexual no hace la menor diferencia, ya que la penetración sexual realizada queda enmarcada en un contexto de ejercicio de poder y no en un ámbito de procura de placer sexual entre dos hombres. Afirmando así que los indígenas Pipiles, al igual que los griegos, no distinguieron entre la homosexualidad y la heterosexualidad (Lara-Martínez, 2012), ya que estos son conceptos acuñados en el siglo XIX. Los Pipiles solamente reconocían a la persona que ejercía el rol receptivo o pasivo designado como cuilonpole/cuilontepole/cuiloni y el tecuilonti como el que ejerce el rol activo, con una clara connotación de jerarquía social de dominador-dominado (Lara-Martínez, 2012).

Con la implementación de las dictaduras militares, los diversos ámbitos de la vida salvadoreña son impregnados por el ejercicio de la violencia homicida. La cultura de dominación se caracterizó por ser machista, mestizo-criolla, blanca, hispanohablante, católica conservadora y heterosexista-normativa. Como punto de quiebre de esa cultura de la dominación, la izquierda

Los Pipiles están relacionados a la cultura Tolteca clásica y fueron el grupo cultural de mayor relevancia a la llegada de los españoles en el actual territorio de El Salvador. Son parte de la macrolengua Yuto-Nahua (Lara-Martínez, 2014). 
política de El Salvador, por medio de la "humillación del otro por su afeminamiento" (LaraMartínez, 2012: 12) utilizan el travestismo como un medio para denunciar las arbitrariedades de los militares y la oligarquía. Su máxima expresión se encuentra en los célebres desfiles bufos de los estudiantes de la Universidad de El Salvador. No obstante, dichas representaciones de género en vez de colocar en la discusión las temáticas de identidad de género y orientación sexual, fueron dispositivos de control y protección que hablan "[...] más sobre las fantasías que genera una cultura heterosexual temerosa para protegerse de sus propias posibilidades homosexuales [...]" (Butler, 2007: 186) y al mismo tiempo cumplen la función de,

\section{[...] suministrar un alivio ritual a la economía heterosexual que debe vigilar constantemente sus propias fronteras contra la invasión de lo "anómalo", y que esta producción y resolución desplazada del pánico homosexual realmente fortalece el régimen heterosexual en su tarea de autoperpetuarse (Butler, 2005: 185).}

Las personas LGBT que existieron en este período se posicionaron con la izquierda política o con las dictaduras militares, no obstante sus prácticas sexuales se mantenían en la marginalidad social. Respecto de este punto, al hablar de marginalidad social se refiere a los espacios de expresión de su sexualidad confinados a lugares que transitan desde lo marginal a lo excluido. Muestra de estos espacios es el mítico salón-bar La Praviana en el centro histórico de San Salvador. Este era reconocido como punto de encuentro de travestis, siendo esta la forma común y denigrante para designar a los hombres que se presentaban con una expresión de género afeminada. Muchos de ellos eran pobres que ejercían la prostitución. Todo ello a pesar de los riesgos y las dificultades de discriminación que se pudieran ejercer sobre ellos.

Otro rasgo característico era la poca presencia de personas jóvenes, los que estaban más ocultos y debido al contexto social de represión, por ello las fiestas privadas en casas particulares eran los espacios de socialización que estos tenían. En dichas fiestas se podía acordar entre los participantes encuentros clandestinos sexuales o la formalización de algún tipo de relación sentimental. Respecto de las mujeres lesbianas no existe ninguna referencia a espacios de socialización conocidos hasta el momento.

En este contexto de polarización social entre los proyectos políticos antagónicos de la derecha militar y la izquierda revolucionaria, el movimiento LGBT no tenía ninguna posibilidad de existencia y visibilidad en la escena política y social. Por el lado militar se observa la lucha contra el comunismo y por la parte de la izquierda se observa la supervivencia e intento de modificar el Estado. Dichas acciones monopolizaban los discursos. Así, la lucha por el poder político se convierte en un metarrelato hegemónico en todos los estratos de la vida nacional. La sexualidad en su más amplio sentido y la homosexualidad en un sentido específico fueron remitidas al silencio político, que colaboró en la perpetuación del heterosexismo.

\section{LA REPRESIÓN POLÍTICA Y ORÁCULOS}

En la década de 1970, en el contexto de la mal Ilamada Guerra Fría entre los bloques capitalista y soviético, El Salvador vive una época de represión política por medio de la implementación de las doctrinas de seguridad interna. Dicha doctrina consideraba a los movimientos sociales organizados de campesinos, estudiantes, sindicatos laborales y los profesores -teniendo a hombres como protagonistas principales-, como el enemigo interno. Estos movimientos sociales inspirados por la victoria de la revolución cubana iniciaron un proceso de organización nunca más visto en la historia reciente del país (Martínez, 2004). 
Las doctrinas de seguridad interna y la organización de los movimientos sociales como era de esperar se confrontan y este choque da como resultado muertes y las desapariciones políticas, estas se convierten en el día a día de la población. Este tipo de acciones intentó erróneamente evitar el aumento de la resistencia política y de las organizaciones de izquierda. No obstante, resultó todo lo contrario, ya que estas se fortalecieron a nivel de simpatía popular y a nivel interno se desarrollaron estrategias para evadir a los cuerpos de seguridad y a los escuadrones de la muerte ${ }^{3}$.

Si es bien cierto que aún no se inicia el movimiento LGBT en esta década, se da un salto cualitativo respecto de los periodos anteriores, ya que se crea un espacio de encuentro gay y travesti por medio de la inauguración de la discoteca Oráculos en San Salvador (Gayelsalvador, 2008). Dicha discoteca surge en 1976, con la clara motivación de crear un lugar de encuentro diferente a los existentes en el centro de San Salvador o en casas particulares. Estuvo ubicada en una central avenida capitalina, facilitando el acceso a personas profesionales que deseaban evitar, probablemente, los riesgos sociales de visitar los puntos de encuentro marginales del centro de San Salvador. En cuanto a las casas particulares muy probablemente ya no proporcionaban lo necesario para realizar actividades de grupos grandes, o por la dificultad de movilización fuera de San Salvador por los retenes militares y los riesgos de supervivencia que existían en esa época.

El surgimiento de la discoteca Oráculos en San Salvador coincide con el fenómeno social de proliferación de grupos sociales antes del estallido de la guerra. Para el caso de la discoteca Oráculos y el sentimiento de pertenencia que esta representaba para su dueño y comensales de este periodo, está muy alejado de los planteamientos de "intereses de clases" que Martín-Baró (2008: 211) menciona como una característica de este periodo histórico en El Salvador. La discoteca más bien era un espacio que brindaba la libertad de expresar la orientación sexual e identidad de género de sus clientes con cierta libertad. Este espacio permite soportar el ambiente hostil de la represión política previo al inicio de la guerra interna salvadoreña que toda la población sufría y a la vez permitía la liberación del peso asfixiante de las normas heterosexistas de la cultura salvadoreña. Oráculos para muchos de sus clientes fue el espacio donde descubrieron su identidad y un sentido de vida inmediato frente a la posibilidad de muerte constante que reinaba en el país.

\section{3. ¿UNA GUERRA INTERNA ÚNICAMENTE DE HETEROSEXUALES?}

Al inicio de la década de 1980 las condiciones sociales y políticas en El Salvador promueven el episodio más oscuro de su historia contemporánea reciente: la Guerra Interna ${ }^{4}$. Esta guerra se inició en 1981 y concluyó en 1992, generando un saldo de 75.000 muertes de personas no relacionadas a los bandos en contienda, así como un millón de personas refugiadas, exiliadas o migrantes y 500.000 desplazados internos (Sue-Montgomery y Wade, 2006: 129;

3 Los escuadrones de la muerte eran grupos organizados clandestinos de los cuerpos de seguridad del Estado que se dedicaban a realizar acciones paralelas o encubiertas del Estado contra la población organizada y civil. La estrategia de los escuadrones de la muerte para disuadir a la población en el involucramiento de las organizaciones sociales era la realización de procesos selectivos de eliminación de personas y la exposición de sus cadáveres con muestras de tortura en vías públicas de constante tráfico como la carretera panamericana que atraviesa el país de occidente a oriente o en espacios naturales como la zona de lava volcánica petrificada del volcán de San Salvador conocida como El Playón.

$4 \quad$ Hago uso del concepto Guerra Interna para no utilizar el apelativo de "guerra civil", en el entendido de que ninguna guerra conlleva a un proceso de civilización o humanización; sino todo lo contrario, es de deshumanización. 
Vásquez, Ibañez y Murguialday, 1996: 91; Navas, 2011: 55). Los 11 años de guerra generaron un desgaste en todos los niveles de la vida cotidiana en El Salvador.

En el contexto machista y heterosexista-normativa, la guerra fue un escenario más para mostrar quién es un hombre de verdad. Se desconoce la existencia de personas LGBT al interior de la guerra interna de forma explícita.

Para encontrar algunas pistas acerca de la participación de personas LGBT en la guerra, recurro a la literatura de denuncia testimonial ${ }^{5}$, esta se produjo entre 1960 y fines de la década de 1980, de las que existen algunas referencias puntuales, expresas e implícitas a lo LGBT. Por ejemplo, encontramos una clara referencia de esta situación en la obra Un día en la vida (Argueta, 2005), en donde los efectivos del ejército de la Fuerza Armada representan una exacerbada masculinidad hegemónica (Connell, 2003), la que podía disponer de los cuerpos y la vida de cualquiera que contrariara su autoridad. Así,

Un día se atrevieron a lo peor. Algo que nos hizo morir: el padre Luna fue encontrado medio muerto en el camino hacia Kilómetro. Le habían dejado la cara desfigurada, con heridas por todas partes. Alguien iba pasando por el lugary vio el hombre desnudo que se lamentaba debajo de un barranco. Le habían metido un palo en el ano y todavía lo tenía alli (Argueta, 2005: 38).

Pasando de la literatura a los hechos que le dieron vida, se tiene conocimiento que $76 \%$ de prisioneros políticos hombres sobrevivientes a los cuerpos de represión reportaron al menos una forma de tortura sexual en el periodo de la guerra (Stemple, 2009), y según el Informe de la Comisión de la Verdad (Organización de las Naciones Unidas, 1993), la violencia sexual antecedía a la muerte de los prisioneros. La liturgia de la violencia debía realizar un acto sexual-poder antes de la eliminación de una persona, ya que "sin la sumisión de los cuerpos por la sexualidad, la soberanía política quedaría truncada" (Lara-Martínez, 2012: 232). O tal vez, las violaciones masculinas ejercidas por parte de miembros del ejército son un posible desahogo de una identidad sexual proscrita sin el riesgo de ser degradado al campo de lo abyecto para quien ejercía el acto sexual de penetración.

Por su parte, en Las mil y una historias de Radio Venceremos (López Vigil, 2006) encontramos nuevamente el carácter travesti del enemigo utilizado por la izquierda política. En este caso, el travestir a alguno de los militares, representantes políticos o personajes de la burguesía era utilizado como táctica militar.

Al entonces jefe del ejército, el general Vides Casanova, le pegamos lo de Tuti Fruti 6 . Este General es uno de los mejores estrategas de las filas del enemigo. Es uno de los escasísimos militares que ha entendido la dinámica de la guerra moderna, su componente político. Pero lo de Tuti Fruti le descontrolaba la vida. Luego fue peor, desde que apareció Lotario, un Negrón que lo mataba de celos y que se lo peleaban entre Tuti Fruti y otro oficial. Fijate qué interesante, Vides Casanova no es maricón. El sí es un tipo todo educado, elegante, de ojos verdes, y para colmo se puso Esmeralda como pseudónimo. O sea, que tenía todo los ingredientes

5 Género literario que emerge para narrar la realidad latinoamericana al interior de las dictaduras militares y de la represión política y social que se manifestó en diferentes formas de violencias. En ella se unen la literatura y la historia con una perspectiva política que rescata la voz de los marginados para evitar su olvido, narrando su propia historia y como tal accediendo a la memoria. La literatura de testimonio se constituye en un contrapeso al monólogo discurso oficial de la historia (André, 2002).

$6 \quad$ Ensalada preparada con diferentes frutas. 
para tildarlo de culero. Y así lo sacábamos una y otra vez en la Guacamaya subversiva', como culero. Y de tanto machacar, hasta los mismos soldaditos se lo acabaron creyendo y miraban con desconfianza a su superior [Cursivas del original] (López Vigil, 2006: 429).

Es factible en este punto resaltar las palabras "Vides Casanova no es maricón", palabras que aluden al nivel de educación, elegancia y el aspecto físico intentando resaltar que no era un hombre afeminado pobre que frecuentaba la zona de la Praviana. Dicha frase nos deja entrever que aquella persona sin nivel educativo, que no tiene elegancia (la que muchas veces es relacionada con las prendas de vestir), y cuya apariencia física se aleja del estereotipo de hombre caucásico podría ser designada como culero, loca, maricón, pipián o una de las 100 formas más que existen en el léxico salvadoreño para referirse desdeñosamente a los "individuos «varones»" que se presumen son homosexuales (Romero, 2009: 72-75).

No obstante, en el período de la guerra, en las Mil y una historias de Radio Venceremos se encuentra una de las primeras referencias explícitas a la vivencia de lo LGBT.

\begin{abstract}
También se respecta la homosexualidad. Mirá a Nando el sastre, que le ha hecho los uniformes a media $B R A Z^{8}$. Nando pedía permiso como todos y se iba a gatear con su amigo. Y nadiele reprochaba que fuera maricón (El único problemita con Nando es cuando te quiere medir el tiro del pantalón con la muy mano... jun momento!), Aquí estaba uno que se quiso llamar Lucha Villa. Venían los cuilios y él se daba riata ${ }^{9}$ como todos. Había lesbianas. ¿Quién no supo de los amores turbulentos entre Trini y no me acuerdo la otra? ¿Y qué? Lo del pluralismo vale también para los corazones (López Vigil, 2006: 488).
\end{abstract}

Este testimonio da cuenta de la existencia de personas homosexuales, lesbianas y hasta trans en las líneas de los frentes armados del Frente Farabundo Martí para la Liberación Nacional (FMLN). Ahora bien, la palabra maricón que en forma general tiene un uso peyorativo, se utiliza previo al comentario explicativo de una posible transgresión en lo sexual a un hombre que se presume es heterosexual y con dicha acción lo degradaría al campo de la abyección. El uso del adjetivo cuilios, derivación del culioni anteriormente descrito, es una calificación para degradar al campo de lo afeminado/travestido a los efectivos de la fuerza armada por parte de los guerrilleros, y como un medio para reafirmar su masculinidad.

"Lo de pluralismo vale también para los corazones", aunque esta frase de forma general da una sensación de inclusión, estamos muy alejados de la realidad. En las líneas del FMLN existía una homofobia explícita. Al fundamentar su accionar político dentro de las corrientes ideológicas del marxismo, leninismo, guevarismo y comunismo (Sue-Montgomery y Wade, 2006: 61) el FMLN, siguiendo la línea argumentativa de Wittig (2010: 40), asume de manera implícita y naturalizada que todos aquellos fenómenos que salieran del marco doctrinario de la Lucha de Clases, en este caso la homosexualidad, son considerados como problemáticas burguesas que tendrían fin con el triunfo de la revolución y la dictadura del proletariado. "Sin embargo, y coherentes en el principio fundamental de la entrega a la lucha revolucionaria, si el homosexual guerrillero demostraba valor y compromiso suficiente y reprimía sus comportamientos en público, podía encontrar cabida en el FMLN" (Vásquez, Ibáñez y Murguialday, 1996: 187).

Nombre de uno de los programas de radio producidos por Radio Venceremos.

Brigada Rafael Arce Zablah.

Expresión para luchar, golpear o pelear. 
Lo anterior acontecía en las áreas rurales de El Salvador, pero al interior de la capital existía otra dinámica, vinculada a los ingresos económicos, que a nivel individual cada persona LGBT poseía. En este caso las travestis están confinadas a sitios marginales y excluidos para poder expresar su identidad de género, recurriendo al ejercicio de la prostitución de calle como medio de sobrevivencia.

En el contexto de violación a los Derechos Humanos continuos en la guerra, esta población excluida no escapó de los cuerpos represivos. En este sentido se tiene como ejemplo el accionar de los miembros del ejército, que llegaban a la zona de La Praviana, y bajo presión y amenazas ejercían violaciones sexuales a las travestis, y además les exigían exorbitantes sumas de dinero para la época a cada una de ellas (aproximadamente 50 dólares). Las travestis que no aceptaban pagar esa cantidad eran sometidas a otros tipos de violencias y agresión, por ejemplo, les cortaban el cabello largo y a otras las llevaban a la base militar, en donde eran sometidas a un proceso de violación colectiva por parte de los soldados. No en pocas ocasiones, varias de estas travestis desaparecieron y otras eran localizadas muertas en la zona conocida como El Playón. En este continuo de agresiones, en 1984 se conoce de un caso paradigmático de la muerte de un grupo de 12 travestis en la segunda Ave. Norte, estas fueron brutalmente violadas y asesinadas. Existió una denuncia ante la Comisión de Derechos Humanos, pero esta fue archivada.

Por su parte, otros hombres que ejercitaban el travestismo que poseían un mejor nivel económico tienen en Oráculos una plataforma para manifestar su identidad de género sin los riesgos que conlleva el estar en la calle. De igual forma, los shows de travestis fueron la táctica para atraer clientes a la discoteca, la que pese a la situación de los primeros años de la guerra, mantuvo un lapso de cierta bonanza económica (Gayelsalvador, 2008). La utilización del travestismo en la discoteca sirvió para crear una cápsula de libertad, de identidad y paz ficticia para sus clientes en cada noche que la discoteca estaba abierta.

En la época de la guerra interna existieron menos espacios para discutir las manifestaciones disidentes de la sexualidad binaria que en la época anterior. En el ejército era imposible realizar esta discusión, por ello se realizaba un ejercicio de poder por medio de las violaciones sexuales que incluían a hombres y travestis. Por su parte, en la filas del FMLN lo LGBT no entraba en las discusiones políticas, muy por el contrario, si una persona lesbiana u homosexual daba muestras públicas de estas tendencias era considerado algo anormal, imposibilitándoles en ocasiones el ascenso a cargos de dirección (Vásquez, Ibáñez y Murguialday, 1994: 267, 271). Además, las personas trans se exponían a mayores o menores riesgos dadas sus condiciones económicas. El principal objetivo de la población salvadoreña en el periodo de la guerra era sobrevivir. Por tal motivo, fue improbable que el movimiento LGBT en El Salvador haya surgido en esa época.

\section{ACUERDOS DE PAZ, POSTGUERRA Y LO LGBT}

En 1992 se firman los Acuerdos de Paz. En ese año y hasta 1994 existía en el país una sensación de que la paz era una realidad, aunque en un sentido de paz negativa (Galtung, 1998). Dicha noción de paz negativa hace referencia, por un lado, a la ausencia del conflicto armado -que llegó a su fin por medio de una negociación política-y, por otro, a la vigencia de condiciones estructurales que promovieron la guerra interna. La supraestructura económica y cultural-machista no fue transformada, sino que solamente fue matizada con tintes de apertura política y una reconciliación nacional que solo queda en acuerdos políticos para 
la transferencia de tierras, becas de estudio para los desmovilizados de ambos bandos, la no participación política de los militares y la participación política libre del FMLN (Organización de las Naciones, 1992).

Se puede afirmar que la organización social de las personas LGBT es un fruto de la postguerra. Un primer colectivo de gays y travestis comenzó a reunirse para la realización de un proyecto de atención y prevención del VIH en la comunidad gay, impulsado por la Fundación Nacional para la Prevención, Educación y Acompañamiento a la Persona VIH/SIDA (Fundasida) en 1992. El inicio de este grupo estuvo bajo la conducción de Wilfredo Valencia, centrándose en la capacitación de métodos de prevención del VIH. El 23 de marzo de 1994 William Hernández y Joaquín Cáceres toman la dirección del grupo (conocidos como Grupo Entre Amigos), otorgándole un carácter más organizativo con el objetivo de promover y defender los Derechos Humanos de la comunidad LGBT. Hasta 1997 las acciones del Grupo están amparadas por Fundasida. En 1998 se abren las oficinas independientes del Grupo, el que comienza a ser reconocido socialmente como Asociación Entre Amigos ${ }^{10}$.

Paralelo a este proceso de organización de travestis y hombres gays, existió un proceso organizativo de las mujeres lesbianas. Luego de finalizada la guerra, las mujeres organizadas al interior del FMLN procuran nuevos espacios políticos para canalizar sus demandas, ya sea al interior del ahora partido político o fuera de este (Navas, 2011: 65-66). En este semillero de nueva rutas, según Ready (2007), existió un grupo de lesbianas salvadoreñas y extranjeras que se reúnen, primero para divertirse y luego para reflexionar acerca de la irrupción de la mujer lesbiana como una identidad política al interior de El Salvador. El nombre con el que fueron conocidas fue La Colectiva lésbica-feminista salvadoreña de la Media Luna y tienen una vida institucional entre 1992 y 1997. Su disolución estuvo marcada por las discrepancias con el movimiento de mujeres y el feminista, estas eran excluidas por el pánico cultural-político del propio movimiento de mujeres y feministas a ser identificadas como lesbianas. Asimismo, la salida del país de las mujeres extranjeras que convocaban en mayor medida las acciones de la colectiva y los vínculos afectivo-políticos que en determinados momentos generaron disputas y rupturas profundas que no permitieron la continuidad de la vida institucional de la colectiva.

Simultáneamente a estos movimientos lésbico y gay organizativos visibles, existió otro denominado Mano Amiga integrado por profesionales gays, grupo cerrado que mantenía en el anonimato a sus miembros. Este grupo, por lo que se conoce, tuvo una vida asociativa de dos años.

En junio de 1997 se realizó la primera Marcha del Orgullo Gay. Por medio de invitaciones fotocopiadas y pegadas en los postes del tendido eléctrico y telefónico del centro histórico de San Salvador se realizaba un Ilamado a participar en la Gran Marcha del Orgullo Gay. La Marcha tuvo un carácter de protesta en recuerdo de la masacre de las 12 travestis por parte del ejército en 1984 y tiene una participación de 250 personas, aproximadamente, sorprendiendo a los propios organizadores que esperaban una participación de 20 a 50 personas. Su recorrido fue desde el parque Cuscatlán hasta la Plaza Francisco Morazán en el centro Histórico de San Salvador. Contó con un apoyo económico puntual de la organización Mano Amiga para suplir algunos de los gastos de su realización. Desde esa fecha hasta la actualidad

10 Para más información www.entreamigoslgbt.org 
año tras año se realiza este evento. La Marcha por la Diversidad Sexual, nombre con que se le conoce actualmente, logró reunir a 6.000 personas en el 2015, según sus organizadores, con representaciones de colectivos y organizaciones LGBT de todo el país.

La Asociación Entre Amigos también desempeñó la función de semillero para la creación de otras organizaciones LGBT. Las nuevas organizaciones LGBT que fueron surgiendo a finales de 1990, la mayoría de sus fundadores pasaron por procesos educativos y formativos de prevención del VIH, Diversidad Sexual y Derechos Humanos que Entre Amigos brindó.

Al interior de Entre Amigos entre 1999 y 2004 existió un considerable componente de mujeres lesbianas, siendo liderado por una de las integrantes de la colectiva Media Luna, pero por situaciones de diferencias y metodologías se desarticuló (Matus, 2011). Muchas de sus integrantes fueron hacia el movimiento feminista. Las fronteras de las organizaciones de mujeres lesbianas son muy permeables, por tal situación estas pueden transitar entre el movimiento lésbico, el feminista y el de mujeres, lo que trae como dificultad su poca visibilidad política, ya que muchas colectivas han surgido, pero de igual forma desaparecieron en esta época.

\section{UN NUEVO MILENIO, LAS MISMAS DIFICULTADES}

El nuevo milenio para la población LGBT se inicia con la fase de expansión de la epidemia del VIH en la población gay y trans, lo que demandó un esfuerzo conjunto con las personas viviendo con VIH para demandar al Estado protección y acceso a los medicamentos. Estas demandas consiguen la distribución gratuita de los antirretrovirales por parte del sistema público de salud por medio de la Ley de Prevención y Control de la Infección provocada por el Virus de Inmunodeficiencia Humana en el 2001.

En el 2000 surge un colectivo denominado Fraternidad Gay sin Fronteras para trabajar con hombres jóvenes en procesos educativos que utilizan el arte y la cultura para sensibilizar y prevenir el VIH-SIDA.

A inicio del 2001 José Francisco García, en calidad de socio fundador de la Asociación para la Libertad Sexual El Nombre de la Rosa, presenta una impugnación por lesividad a sus derechos constitucionales de igualdad y libre asociación, ante la Corte Suprema de Justicia (CJS), originada por la negativa de concesión de la personería jurídica por parte del Director General del Registro de Asociaciones y Fundaciones Sin Fines de Lucro del Ministerio de Gobernación. Este manifestaba que dicha Asociación,

[...] violentaban normas de derecho natural, los fines que persigue la familia, la constitución del matrimonio, las buenas costumbres, la moral y el orden público; como también porque lo pretendido por la entidad en formación iba en contra de los principios establecidos en el art. 3 inc. $2^{\circ}$ de la Ley de Asociaciones y Fundaciones Sin Fines de Lucro (LAFSFL), el cual establece que los fines de las asociaciones y/o fundaciones no deben contrariar "el orden público, la moral, la ley y las buenas costumbres" (Corte Suprema de Justicia, 2009: 1).

En el 2003 las fracciones legislativas conservadoras de derecha y fundamentalista evangélicas aprueban una solicitud de reforma de la Constitución Política presentada por el Partido Demócrata Cristiano, la que plantea lo siguiente:

Art. 32: [...] Serán hábiles para contraer matrimonio entre ellos el hombre y la mujer, así nacidos [... Los matrimonios entre personas del mismo sexo celebrado o reconocidos bajo 
las leyes de otros países, y otras uniones que no cumplan con las condiciones establecidas por el orden jurídico salvadoreño no surtirán efecto en El Salvador.

Art. 33: [... Regulará asimismo las relaciones resultantes de la unión estable de un hombre y una mujer, así nacidos y que no tengan impedimento para contraer matrimonio.

Art. 34: [...] Se prohíbe la adopción por parejas del mismo sexo (Ayala, 2009: 7-8).

Esta reforma intenta cerrar la posibilidad de que personas del mismo sexo puedan ejercer el derecho de la unión civil y la adopción. Para que esta reforma sea efectiva, son necesarias dos votaciones en periodo legislativos diferentes. En el 2003 se logra la primera votación. En el 2006 esta reforma constitucional no alcanza los votos necesarios para su ratificación, por lo que es archivada.

A lo largo de esta década la Asociación Entre Amigos sufre ataques, espionaje telefónico y saqueos de materiales documentales en las oficinas. En 1999 William Hernández recibió amenazas de muerte, por ser la primera figura pública que asumía su condición de bisexual y representante del movimiento gay en El Salvador. Por medio de una campaña de presión política acompañada por Amnistía Internacional William obtuvo protección policial desde 1999 hasta el 2007 (Asociación “Entre Amigos", 2010).

El Concejo Municipal de San Salvador (2006) se proclama por los derechos humanos de las diversas identidades sexuales de la ciudad de San Salvador. En esta declaración se hace un llamado por el derecho de ciudadanía, el aporte de las personas LGBT en diferentes ámbitos de la capital, la no discriminación por cualquier tipo de marcador social diferenciador incluyendo la condición sexual o identidad de género de los habitantes de la ciudad.

En el 2007 la Asociación Entre Amigos presenta una petición ante la Asamblea Legislativa para declarar el 17 de mayo como Día Nacional contra la Homofobia. El pensamiento conservador tergiversa esa propuesta y mediáticamente se expone que esa propuesta estaba promoviendo la homosexualidad. Con esta base mediática el Partido Demócrata Cristiano retoma nuevamente el tema de las reformas constitucionales. Acontece un momento crítico con las campañas realizadas por la Iglesia católica y los grupos conservadores, que propiciaron el aumento de los crímenes de odio homofóbico. Estos crímenes, con una violencia cuasibarbárica dirigidos a la población LGBT, marcan su punto más elevado en junio de 2009, donde se reportan alrededor de 10 casos registrados en ese mes y 27 en el año (Asociación "Entre Amigos", 2010: 11). El caso paradigmático es la muerte de Tania, adolescente trans que fuera secuestrada de su lugar de trabajo, torturada por siete días y su cadáver fue expuesto en una importante vía pública (Ayala, 2009,15-16), al mejor estilo de los escuadrones de la muerte antes y durante la guerra interna.

El movimiento lésbico, en el final de la década del 2010, estructura colectivas de vida institucional mayor, a diferencia de la década pasada. Colectivos como Las Desclosetadas, el Movimiento Lésbico Juvenil -Lesbos- y el Espacio de Mujeres Lesbianas -Esmules- comienzan su trabajo organizativo en 2007, 2008 y 2009, respectivamente. El movimiento de mujeres lesbianas tiene una clara distinción respecto del movimiento gay o trans. El trabajo acerca de VIH, que ha sido el foco principal de los anteriores, no es hegemónico en el movimiento lésbico, por lo que sus estrategias de incidencia política y liberación del cuerpo de las mujeres presentan una diversificación de caminos. Por ejemplo, el arte, la comunicación 
o la investigación se muestran como ejes institucionales para presentar sus demandas y reivindicaciones a la sociedad salvadoreña.

Las mujeres trans inician un trabajo organizativo más visible en esta década también, existiendo una separación del movimiento de hombres gays. A nivel de ejemplo retomaremos el trabajo realizado por Karla Avelar de Concavis-Trans ${ }^{11}$, para relatar el proceso organizativo de las mujeres trans. Esta organización nace debido a las condiciones de discriminación que las mujeres trans padecen y en específico las mujeres trans viviendo con VIH. Su constitución se dio por medio de la creación de un grupo de apoyo exclusivo para mujeres trans viviendo con VIH para evitar la discriminación y culpabilización que personas integrantes de los grupos de apoyo de personas viviendo con VIH realizaban hacia ellas. Su inicio tuvo muchas dificultades por la desinformación existente, la falta de local y capacidades organizativas, mas contaron con el apoyo de la Asociación Entre Amigos en este inicio. Cuando su trabajo comenzó a tomar forma, la idea de tener un grupo de apoyo resultó ser insuficiente, ya que lo que se necesitaba era una organización que defendiera y promoviera el cumplimiento de los Derechos Humanos de las mujeres trans viviendo con VIH o no, en los espacios médico-hospitalarios en específico y la sociedad en general, para minimizar la poliédrica discriminación de ser trans, ejercer la prostitución, ser pobre, expresidiaria en algunas ocasiones y convivir con VIH en otras.

En el 2009 fue colocada nuevamente la reforma constitucional en la agenda legislativa de los partidos políticos conservadores. Los promotores de tal reforma esperaban que su aprobación fuera un procedimiento de trámite legislativo nuevamente como en el 2003, pero aconteció exactamente lo contrario. Las organizaciones LGBT existentes conscientes que la reforma constitucional no radicaba exclusivamente en la prohibición de establecer una familia homoparental, sino que esta legalizaría la invisibilidad de los Derechos Constitucionales de las minorías sexuales, crean la Alianza para la Diversidad Sexual, unificando a las diferentes organizaciones LGBT, de prevención del VIH y de activistas independientes ejerciendo un contrapeso para debatir, confrontar, exponer y proponer ante la Asamblea Legislativa y la sociedad en general una alternativa a tal reforma. En esta oportunidad se presenta y vota una nueva versión de tal reforma, que en nada modifica su sentido discriminatorio (Ayala, 2009).

Un hecho relevante a finales del 2009 fue la resolución de la Corte Suprema de Justicia (CSJ) a la impugnación de la negativa de otorgamiento de personería jurídica por parte del Ministerio de Gobernación a la Asociación para la Libertad Sexual El Nombre de la Rosa. La CSJ declara ha lugar el amparo solicitado contra la actuación del Director General del Registro de Asociaciones y Fundaciones Sin Fines de Lucro del Ministerio de Gobernación, reconociendo la violación de los derechos de libre asociación e igualdad (Corte Suprema de Justicia, 2009) que este había ejercido contra esta y otras organizaciones LGBT. Para este momento, la Asociación El Nombre de la Rosa ya no tenía vida institucional, pero quedará para la historia su solicitud y la resolución que benefició a las organizaciones LGBT en funcionamiento en esa época hasta la actualidad.

\section{CONQUISTAS POLÍTICAS E INTERNACIONALIZACIÓN}

El 2010 se inicia con el cambio político ocurrido un año antes. El otrora movimiento revolucionario de la década de 1980, el FMLN, ocupa la conducción del Órgano Ejecutivo, posibilitando

11 Para más información www.comcavis.org.sv 
una apertura histórica a las personas LGBT para discutir temas respecto de identidad de género y orientación sexual, y sobre todo la disponibilidad de acceso a los espacios de toma de decisiones de políticas públicas (Ayala, 2009). En este sentido se crea una plataforma mínima LGBT para que el Gobierno Central que asume el cargo ejecutivo en sus 5 años de gobierno.

En el 2010, gracias a la resolución de la CSJ acerca de la obtención de personería jurídica de asociaciones LGBT, la obtención del estatus legal se da bajo los criterios establecidos por la ley sin discriminación por orientación sexual o identidad de género. Esto produjo, para ejemplificar con la Asociación Entre Amigos que por más de 14 años y tres negativas institucionales para su registro legal, después de esta resolución y el cambio político, en tres meses concluyera el proceso de registro (Asociación "Entre Amigos", 2010).

La Presidencia de la República adoptó el Decreto No 56 (2010), que establece disposiciones para evitar toda forma de discriminación en la Administración Pública por razones de identidad de género o de orientación sexual.

En mayo de 2010, con el apoyo de las organizaciones LGBT, el Estado creó la División de Diversidad Sexual, bajo los auspicios de la Secretaría de Inclusión Social. Su trabajo básicamente ha consistido en la asistencia, capacitación y sensibilización en Derechos Humanos, Género y Diversidad Sexual. Su foco han sido las áreas de educación, trabajo, salud, acceso a la justicia, seguridad y recreación para la población LGBT. Como un proceso incipiente de políticas públicas este trabajo no está exento de críticas. Las organizaciones LGBT esperaban una transformación radical del ejecutivo respecto de los temas LGBT, pero la realidad ha sido la apertura de pequeños espacios para (re)conocer los Derechos Humanos de las personas LGBT en las instancias gubernamentales.

El 17 de mayo de 2010 las organizaciones trans convocan a la primera marcha contra la homo y transfobia. Su punto de partida fue la Plaza Las Américas, conocida como El Salvador del Mundo hasta el monumento a la Constitución ${ }^{12}$ evidenciándose su nivel organizativo y su capacidad de convocatoria política. Su recorrido fue extremamente simbólico, ya que parte desde el icono de representación de lo salvadoreño: El Salvador del Mundo (López Bernal, 2011), hasta el monumento de la Constitución que de forma alegórica representa la Justicia. En otras palabras, son salvadoreños/as que demandan justicia. En esta primera oportunidad las organizaciones trans vistieron al monumento de la Constitución con la bandera del arcoíris. Esta fuerza organizativa de las mujeres trans también se ha volcado a la creación de una Ley sobre Identidad de Género, esto crea en cierto momento una división con otras organizaciones gay que promulgan la necesidad más que una Ley de Identidad de Género, la creación de una ley de no discriminación por orientación sexual e identidad de género.

El 17 de mayo de 2012 se conforma al interior de la Procuraduría para la Defensa de Derechos Humanos (PDDH), la Mesa Permanente sobre Derechos Humanos de la población LGBT (Davenport, 2012: 22). El objetivo general de la Mesa es incidir para que la población LGBT tenga acceso al pleno goce de sus derechos humanos, además de llevar a cabo otras acciones tales como: analizar la situación de la población LGBTI en El Salvador, establecer e implementar los procesos de sensibilización para los funcionarios encargados de la

12 Conocida coloquialmente como la chulona, que es una expresión salvadoreña para designar a una mujer desnuda, y a un hombre se puede referir como el chulón. Por tal motivo, las organizaciones trans colocaron en forma de ropa la bandera del arcoíris en la referida estatua. 
administración de justicia hacia dicha población, establecer las bases para la elaboración de una ley para la erradicación de cualquier forma de discriminación a la población LGBTI, entre sus apuestas más importantes (Procuraduría para la Defensa de los Derechos Humanos, 2012).

En junio de 2012 se realiza la primera Plegaria Rosa LGBTI en el monumento de El Salvador del Mundo como un acto para recordar a las personas LGBTI víctimas de los crímenes de odio por orientación sexual e identidad de género. Esta gestión del duelo colectivo, utilizando las palabras de Butler (2010), permite que una vida precaria LGBT por la discriminación y el consecuente crimen de odio se transforme en una vida digna para ser llorada y recordada en un duelo.

A nivel nacional e internacional, las diferentes organizaciones LGBT conforman alianzas para promover la defensa de los Derechos Humanos de la población LGBT. Toda esta sinergia institucional promovió la realización de estudios e investigaciones acerca de la situación de los Derechos Humanos de poblaciones $\mathrm{LGBT}^{13}$. En este punto se debe resaltar que por primera vez en el Informe de Desarrollo Humano El Salvador 2013 se explicita la orientación sexual como dificultad para obtener empleo, tratamiento diferenciado para alcanzar un bienestar de vida al interior de El Salvador y condición humana que no debe generar actos de discriminación (PNUD, 2013: xvii,19,167).

En el 4 de mayo de 2013 es asesinada, tras múltiples amenazas de muerte por parte de supuestos pandilleros, la activista Tania Vásquez, de Comcavis-trans. En el juego perverso que interactúa la invisibilidad y negación de derechos, los agentes institucionales reproducen el modelo de violencia a la población LGBT,

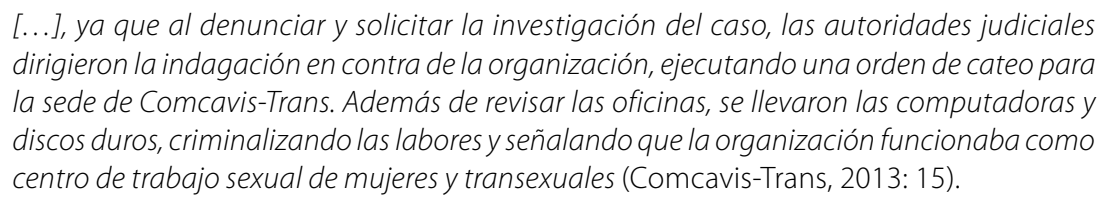

Debido a esta situación, a pesar de no contar con información, experiencia y desconocimiento de los procesos para presentar una solicitud de audiencia ante la Comisión Interamericana de Derechos Humanos, Karla Avelar realiza dicha solitud en donde requirió audiencia para presentar la situación de violencia contra la población de mujeres trans en El Salvador. La audiencia es aprobada para presentar su informe el 29 de octubre de 2013. Ante esta aprobación, Karla Avelar visualiza la necesidad de involucrar a otras organizaciones trans en este proceso, ya que no era una solicitud a título personal, sino que era una exposición de la situación del colectivo de mujeres trans en El Salvador.

En noviembre de 2013 un conocido presentador de televisión, en su blog personal, publicó un escrito titulado "Una visita a la casa de Fabián y Tulio". En él presentó una homofóbica visión de la convivencia cotidiana entre una pareja integrada por mujeres. Dicha publicación

13 Situación de población adulta mayor LGBT en El Salvador (2015). Violaciones de Derechos Humanos de las Personas Transgénero de El Salvador (2015). Informe de El Salvador sobre Derechos Humanos de las Personas Trans (2014). The Global Divide on Homosexuality (2013). Sondeo de Percepción de los Derechos Humanos de la Población LGBTI de EI Salvador (2013). Diagnóstico jurídico sobre Derechos Humanos de la Población LGBTI de El Salvador (2012). Diversidad Sexual en El Salvador (2012). La situación de los Derechos Humanos de las Personas Lesbianas, Gays, Bisexuales y Transgénero en El Salvador (2010). 
ocasionó una revolución en las redes sociales, lo que obligó en un primer momento a cerrar dicho blog. Luego de las manifestaciones públicas de activistas LGBT en los estudios de producción de su programa de televisión, dicho presentador solicitó disculpas por los daños causados, reconociendo su desconocimiento referente a dicha temática y solicitando la documentación necesaria para incrementar su conocimiento de la población LGBT y mejorar su nivel cultural al respecto de dicho tema (Serrano, 2013).

Al interior de la Universidad de El Salvador en el 2013 surge un primer colectivo LGBT denominado Diversidad UES, promovido por estudiantes de la Facultad de Medicina. Su trabajo inicial al interior de la Alma Mater es extremadamente simbólico. Por ejemplo, en mayo de 2014 realizaron el primer conversatorio acerca de Diversidad Sexual en el auditorio de la Facultad de Derecho, el mismo recinto que vio nacer al FMLN en octubre de 1980. En noviembre de 2014 izaron la bandera del arcoíris en la Plaza Minerva y se realizó el primer conversatorio de Diversidad Sexual con representantes estudiantiles de otras universidades del área metropolitana.

El 11 de enero de 2014 se conforma un primer colectivo de Hombres Trans denominándose Hombres Trans de El Salvador o conocidos como HT503. El colectivo surge por la necesidad de visibilizar la expresión de género diferenciada al movimiento de mujeres lesbianas, y al mismo tiempo como un paso para visibilizar esa identidad en la palestra nacional. Este colectivo ha tenido el apoyo de Comcavis-trans para uso de su local para realizar reuniones de formación, información y fortalecimiento institucional con vistas a la obtención de su personería jurídica. Una de sus principales apuestas como colectivo es la discusión de la masculinidad hegemónica, para no reproducir estos mismos patrones en la construcción de la masculinidad de sus miembros. El colectivo está articulándose con otras organizaciones de hombres trans a nivel de Centroamérica, lo que marca un proceso diferenciador a los anteriores movimientos lésbicos, gays o de mujeres trans que se organizaron de forma solitaria.

Debido al trabajo realizado por las diversas organizaciones LGBT, logran un éxito en el 2014 ante el Tribunal Supremo Electoral (TSE), para que este gire la orden del reconocimiento del derecho al voto por parte de las personas trans, aunque su imagen del Documento Único de Identidad (DUI) reflejase una identidad de género diferente a la actual. Al mismo tiempo, las organizaciones LGBT se organizan para ejercer la función de observadores nacionales en el proceso electoral. En la primera votación presidencial del 2 de febrero de 2014 se acreditó a 17 observadores LGBT distribuidos en 5 municipios de El Salvador (Aspidh Arcoíris, 2014). En la segunda vuelta para elegir al Presidente 37 observadores LGBT se desplazaron a 23 municipios el 9 de marzo de 2014 (Cisneros, 2014). En ambos procesos electorales los observadores registraron que la burla, la risa y las miradas de crítica son los actos de discriminación al momento que las personas LGBT ejercen su derecho al voto (Aspidh Arcoiris, 2014; Cisneros, 2014).

En el 2015 se celebraron las elecciones para alcaldes y diputados, y en esta oportunidad aparece una primera candidatura para diputado suplente de una persona LGBT. Un partido conservador en busca de nuevos cotos de votos presenta como candidato de diputado suplente a un hombre trans. Por medio del eslogan Hagamos la diferencia sin ser diferentes, pretendió ser una candidatura representativa de la comunidad LGBT, mas tal situación no progresó.

Luego de esa elección parlamentaria, el 15 de abril de 2015 la Asamblea Legislativa vuelve a aprobar la reforma constitucional de los Arts. 32, 33 y 34. Ahora esta reforma se encuentra a la espera de su ratificación por mayoría calificada en el periodo legislativo 20152018, aguardando el momento oportuno para que los partidos políticos conservadores la 
conviertan en una moneda de cambio por otros asuntos de interés político. El 14 de mayo de 2015 los diferentes colectivos y organizaciones LGBT presentan ante la Corte Suprema de Justicia un recurso de amparo de inconstitucionalidad, asumiendo que la reforma aprobada de los artículos 32, 33 y 34 de la Constitución, lacera el principio de Igualdad en las personas salvadoreñas LGBT, establecido en el artículo 3 de la Constitución.

\section{A MANERA DE CIERRE}

El movimiento LGBT es una realidad. Hoy nadie puede ignorar la existencia de un movimiento social que vela por los Derechos Humanos de las personas LGBT. En 20 años de estructuración se han organizado hombres gays, mujeres trans, lesbianas y hasta hace pocos años hombres trans y jóvenes LGBT a nivel de la capital. El cuestionamiento de los patrones binarios de la sexualidad y los esencialismos de género, por parte de las personas LGBT organizadas, ha promovido diferentes estrategias de visibilidad y acciones políticas.

Respecto de la organización política de las identidades LGBT podemos proponer cuatro momentos u "Olas", utilizando las palabras de los antropólogos brasileños Simões y Facchini (2009) al referirse al movimiento LGBT de Brasil. La Primera Ola comprendería el periodo antes de 1992, caracterizándose por la "invisibilidad" social de la homosexualidad, el surgimiento de la discoteca Oráculos como un espacio de sociabilidad homosexual. No obstante, las condiciones sociales y políticas del país no fueron las idóneas para el surgimiento de una organización política LGBT.

La Segunda Ola, entre 1992 y 1999, se puede llamar como "Power Gay", retomando el eslogan de la primera marcha LGBT realizada en San Salvador en 1997. Entre las características más importantes que se pueden resaltar se encuentra la organización de colectivos y grupos gays, que incluían a mujeres trans, y la existencia de un colectivo lésbico de corta duración. El mayor logro del movimiento LGBT incipiente en este periodo fue el de poder sobrevivir a todas las dificultades sociales, políticas, organizacionales que existieron.

La Tercera Ola, entre 2000 a 2008, la podemos nombrar como "LGBT". La principal característica en este periodo histórico fue la diversificación del propio movimiento. Así podemos observar la separación de hombres gays y mujeres trans, denotando el surgimiento de la identidad política trans con este hecho. Aunque continuaron compartiendo el facto que el $\mathrm{VIH}$ era uno de sus principales ejes articuladores tanto para las identidades gays como trans. Las colectivas de mujeres lesbianas en esta época se consolidaron.

La Cuarta Ola, de 2009 hasta la fecha, se puede denominar como "Diversidad Sexual". Su principal característica es el surgimiento del movimiento LGBT como actor político en la palestra nacional, el que realiza demandas a la agenda pública del gobierno de respeto de los Derechos Humanos de las personas LGBT. En este periodo se promueve una visibilidad internacional de las demandas de respeto y protección de los Derechos Humanos y acceso a la justicia para esclarecer los casos de violencia homofóbica que afectan a todas las identidades LGBT, pero con especial incidencia en las identidades trans, tanto femeninas como masculinas. También en este periodo se presenta una apertura del Ejecutivo para abordar cuestiones de orientación sexual e identidad de género, esto ha promovido acciones para cierto reconocimiento institucional, como lo fue el Decreto Ejecutivo No 56 de 2010, la creación de la Dirección de Diversidad Sexual en 2010, reconocimiento del derecho al voto en 2014, la legalización de sus organizaciones y la creación de la Mesa Permanente sobre Derechos Humanos de la población LGBTI al interior de la Procuraduría para la Defensa de los Derechos Humanos en 2012. 
No obstante, a pesar de las conquistas políticas restringidas obtenidas por parte del movimiento LGBT, aún hace falta mucho para la transformación de las políticas y discursos públicos existentes impregnados por el heterosexismo normativo que naturalizan la homofobia, y promueven colateralmente los crímenes de odio contra personas LGBT.

Una de las limitaciones del movimiento LGBT es la centralidad geográfica que este tiene en San Salvador. Los mayores actos de visibilidad y demanda de reconocimiento se efectúan a nivel de la capital. No obstante, se reconoce que han existido y existen colectivos en otros departamentos de El Salvador, pero su vida institucional es poco conocida. La organización o fortalecimiento de otros colectivos LGBT fuera de San Salvador es un reto para el propio movimiento. Esta historia también es necesaria conocerla y resguardarla.

En el contexto del patriarcado en el siglo XXI, el movimiento LGBT en El Salvador, diverso en sus orígenes y mucho más en sus expresiones, tiene como uno de sus retos enfocarse en la creación de estrategias políticas internas y externas para canalizar de mejor manera sus energías y luchas. Estas estrategias políticas deberían permitir, en un futuro próximo, la representación de personas LGBT en cargos de elección popular como alcaldías y la Asamblea Legislativa, en calidad de propietarios y no únicamente de suplentes.

A pesar de los avances políticos y sociales de las personas LGBT en otros países de América Latina, en El Salvador, la invisibilidad, la negación, el rechazo institucional hasta cierto reconocimiento restringido, se podría caracterizar la forma cómo el Estado ha tratado a las identidades LGBT en los periodos analizados. La invisibilidad de estas identidades ha sido posiblemente la norma tácita para no dar atención a las personas LGBT. Cuando tales identidades están visibles, a estas se les intentan negar sus derechos, como el caso de la legalización de sus asociaciones y organizaciones en las dependencias correspondientes del Estado.

Este artículo es un primer boceto, inacabado, de la organización social y política del movimiento LGBT. Aunque la idea inicial era la construcción de un panorama general de la organización del movimiento LGBT en El Salvador, en más de uno de sus apartados no pasó de ser una crónica que recopila acciones que los colectivos, asociaciones y organizaciones LGBT han realizado hasta la fecha. Inacabado, ya que al momento de ser escrito la historia misma del movimiento continúa aconteciendo, y a cada mes y a cada año existen nuevas informaciones para ser registradas.

Entre las deudas pendientes que deja este artículo, pero que al mismo tiempo se pueden transformar en propuestas para nuevas investigaciones se encuentran: el análisis del movimiento LGBT desde una perspectiva interseccional (Piscitelli, 2008) que aborde las categorías de género, generación, raza-etnia, clase social y geografía, entre las más sobresalientes, para comprender la interacción entre las múltiples formas de subordinación, opresiones, negociaciones y adaptaciones que atraviesan al propio movimiento y a las identidades políticas LGBT que lo integran. El mercado de consumo, los locales de comercio y sus relaciones con la organización social LGBT también es una temática de interés para ser investigada, para corroborar si las palabras del sociólogo francés Frédéric Martel se cumplen en El Salvador: "la liberación gay empieza a menudo con los bares y los clubs, es decir, con el comercio y el mercado" (2014: 20). El estudio de la migración de las personas LGBT debido al rechazo de su orientación sexual y expresiones de género, es un tema que no ha sido lo suficientemente investigado. El análisis de la política sexual (Carrara, 2010) al interior de El Salvador y sus interrelaciones con el movimiento LGBT es otra temática para ser abordada. Para un posterior estudio se encuentra establecer un diálogo comparativo entre países de América Latina y El Salvador, respecto de la organización y politización de identidades LGBT. 
Para finalizar, se reconoce que la construcción de la memoria histórica del movimiento LGBT no puede ser hecha únicamente por una persona, se requiere un esfuerzo colectivo, que incluya actores del movimiento social y académicos, tanto adentro de El Salvador como afuera del país, para impedir que el silencio que ha sido cómplice del tabú y este de los homicidios promovidos por la homofobia, no cobre más vidas de personas LGBT.

\section{BIBLIOGRAFÍA}

André, R. (2002): El testimonio, Roque Dalton y la representación de la catástrofe [Tesis Doctoral]. São Paulo: Universidade de São Paulo.

Argueta, M. (2005): Un día en la vida. San Salvador. Dirección de Publicaciones e Impresos.

Audiovisuales de la UCA (2007): 1932, la negación indígena [Documental], San Salvador: UCA.

Asociación Comunicando y Capacitando a Mujeres Trans con VIH en El Salvador (2013): Informe para la Audiencia ante la Comisión Interamericana de Derechos Humanos sobre la situación de violencia contra la población de mujeres trans en El Salvador. San Salvador: Comcavis-Trans.

Asociación Salvadoreña de Derechos Humanos "Entre Amigos" (2010): La situación de los Derechos Humanos de las personas lesbianas, Gays, Bisexuales y Transgénero en El Salvador. Informe Alterno sometido al Comité de Derechos Humanos de las Naciones Unidas. San Salvador: Asociación "Entres Amigos".

Asociación Salvadoreña de Derechos Humanos "Entre Amigos" (2010): La situación de los Derechos Humanos de las personas lesbianas, Gays, Bisexuales y Transgénero en El Salvador. Informe Alterno sometido al Comité de Derechos Humanos de las Naciones Unidas. San Salvador: Entre Amigos.

Asociación Solidaria para Impulsar el Desarrollo Humano (Aspidh-Arcoíris) (2014): Informe de observación electoral presidencial -2 de febrero 2014-. San Salvador: Aspidh-Arcoíris.

Ayala, A. (2009): Sistematización de hechos de agresión a la comunidad de lesbianas, gays, bisexuales y trans de El Salvador. San Salvador: Alianza por la Diversidad Sexual LGBT.

Barrientos, J. (2015): Violencia homofóbica en América Latina y Chile. Santiago de Chile: Ediciones y Publicaciones El Buen Aire.

Bourdieu, P. (1999): A dominação masculina. Rio de Janeiro: Bertrand Brasil.

Butler, J. (2005): Cuerpos que importan: sobre los límites materiales y discursivos del "sexo". Buenos Aires: Paidós.

- (2007): El género en disputa. El feminismo y la subversión de la identidad. L'Hospitalet de Llobregat (Barcelona): Paidós.

(2010): Marcos de guerra. Las vidas Iloradas. Buenos Aires: Paidós.

Carrara, S. (2010): "Políticas e direitos sexuais no Brasil contemporáneo", en Bagoas: Revista de Estudos Gays, Vol. 4, pp. 131-148. 
Cisneros, A. (2014): Informe de Observación electoral LGBTI. San Salvador: Sin editorial.

Connell, R. (2003): Masculinidades. México D. F.: Universidad Nacional Autónoma de México.

Consejo Municipal de San Salvador (2006, junio 22): "Declaración", CoLatino, p. 11, San Salvador.

Corte Suprema de Justicia, Sala de lo Constitucional (2009): 18-2004. San Salvador: CSJ.

Davenport, A. (2012): Diversidad Sexual en El Salvador. Un informe sobre la situación de los Derechos Humanos de la comunidad LGBT. San Francisco: Berkeley Law.

Foucault, M. (1972): A arqueologia do saber. Petrópolis: Editora Vozes.

Galtung, J. (1998): Tras la violencia, 3R: reconstrucción, reconciliación, resolución. Afrontando los efectos visibles e invisibles de la guerra y la violencia. Gernika: Gernika Gogoratuz.

Gayelsalvador (2008): La historia de Oráculos. Disponible en www.gayelsalvador.com/oráculos.htm

International Lesbian, Gay, Bisexual, Trans and Intersex Association (2015): State Sponsored Homophobia 2015: A world survey of laws: criminalisation, protection and recognition of same-sex love. Geneva: ILGA.

Lara-Martínez, R. (2014): Mitos en la lengua materna de los Pipiles de Izalco en El Salvador. Soyapango: Editorial Universidad Don Bosco.

(2012): Indígena, cuerpo y sexualidad en la literatura salvadoreña. Soyapango: Editorial Universidad Don Bosco.

López Bernal, C. (2011): Mármoles, clarines y bronces. Fiestas cívico-religiosas en El Salvador, siglos XIX y XX. Soyapango: Editorial Universidad Don Bosco.

López Vigil, J. (2006): Las mil y una historias de Radio Venceremos. San Salvador: UCA Editores.

Martel, F. (2014): Global gay. Cómo la revolución gay está cambiando el mundo. Lima:Taurus.

Martín-Baró, I. (2012): Acción e ideología: psicología social desde Centroamérica. 15a reimp. San Salvador: UCA Editores.

(2008): Sistema, grupo y poder: psicología social desde Centroamérica (II). $5^{\text {a reimp. }}$ San Salvador: UCA Editores.

Martínez, O. (2004): El Salvador, del Conflicto Armado a la Negociación 1979-1989. San Salvador: Editorial Nuevo Enfoque.

Matus, S. (2011): "Rebeldía, transgresión, persistencia y resistencia. La lucha por los Derechos Humanos de Las Lesbianas en El Salvador", en La Otredad. Disponible en http://mujeresycambio.blogspot.com.br/2011/11/rebeldia-transgresion-persistencia-y.html 
Navas, M. (2011): "De guerrilleras a feministas: elementos para estudiar las organizaciones de mujeres en El Salvador. 1992-1995", en Cultura, № 106, Abr/Jun, pp. 55-68.

Organización de las Naciones Unidas (1992): Acuerdos de Paz de El Salvador. New York: ONU. (1993): Informe de la Comisión de la Verdad. De la locura a la esperanza. La guerra de 12 años en El Salvador. Nueva York: ONU.

Piscitelli, A. (2008): "Interseccionalidades, categorias de articulação e experiências de migrantes brasileiras", en Sociedade e Cultura, 11, № 2, pp. 263-274.

Presidencia de la República de El Salvador (2010): Decreto Ejecutivo No 56. San Salvador: Diario Oficial.

Procuraduría para la Defensa de los Derechos Humanos (PDDH) (2012):"PDDH lanza Mesa Permanente sobreDerechos Humanos de laPoblación LGBTI".'Disponibleen http://www.pddh.gob.sv/menupress/ menunoti/316-pddh-lanza-mesa-permanente-sobre-derechos-humanos-de-la-poblacion-lgbti

Programa de las Naciones Unidas para el Desarrollo (PNUD) (2013): Informe sobre Desarrollo Humano El Salvador 2013. Imaginar un nuevo país. Hacerlo posible: Diagnóstico y propuesta. San Salvador: Impresos Múltiples.

Prudhomme, O. (2010): "Historias pioneras, Fuentes Inéditas. Del carácter pionero de las tesis de licenciatura en Historia escritas en El Salvador", en Identidades, Año 1, No 1, pp. 92-103.

Ready, K. (2007): "The Uneasy "Informal Consensual Union" of Lesbianism with Feminism in El Salvador", en Culture, Health \& Sexuality, 9, Suplemento 1, p. S7.

Romero, M. (2009): Léxico del Cuerpo Humano en el habla popular salvadoreña. San Salvador: Editorial Delgado.

Serrano, I. (2013): "Daniel Rucks se disculpa con comunidad gay: No era mi intención”, en La Página, disponible en http://www.lapagina.com.sv/nacionales/89882/2013/11/21/ Daniel-Rucks-se-disculpa-con-comunidad-gay-No-era-mi-intencion

Simões, J. \& Fachinni, R. (2009): Na trilha do arco-íris. Do movimento homossexual ao LGBT. São Paulo: Editora Fundação Perseu Abrano.

Stemple, L. (2009): "Male rape and Human Rights", en Hastings Law Journal, Vol. 60, pp. 605-647.

Sue-Montgomery, T. y Wade, C. (2006): A revolução salvadorenha: da revolução à reforma. São Paulo: Editora da UNESP.

Vázquez, N., Ibánez, C. y Murguialday, C. (1996): Mujeres Montaña. Vivencias de guerrilleras y colaboradoras del FMLN. San Cristóbal: Horas y Horas.

Wittig, M. (2010): El Pensamiento Heterosexual y otros ensayos. Móstoles: Top Printer Plus. 